Results We included 74 studies from 16 countries comprising 34,535 SSL. Compared with HP, SSL was more likely to be in the right colon (OR 5.45; 95\% CI 4.13-7.17; $\mathrm{P}<0.01$ ); larger in size $(>5 \mathrm{~mm}$ vs. $<=5 \mathrm{~mm}$ OR 5.60 ; 95\% CI $3.82-$ 8.22; $\mathrm{P}<0.01$ ); in Paris-0-II morphology (OR 2.33; 95\%CI $1.45-3.70 ; \mathrm{P}<0.01$ ); having mucus cap (OR 8.48; 95\% CI 4.86-14.8; $\mathrm{P}<0.01)$; vague margin under white light endoscopy (OR 2.71; 95\% CI 1.88-3.92; $\mathrm{P}<0.01)$; having expended crypt opening (OR 5.09; 95\% CI 1.87-13.9; $\mathrm{P}<0.01$ ), varicose microvascular vessels (OR 6.17; 95\% CI 1.57-24.3; $\mathrm{P}<0.01)$ and thick branched vessel (OR 5.17; 95\% CI 1.81-15.8; $\mathrm{P}<0.01)$ under magnified narrow band imaging and type II-O pit pattern (OR 18.56; 95\% CI 8.45-40.7; $\mathrm{P}<0.01$ ) under magnified chromoendoscopy.

Conclusions We systematically synthesised current evidence on the discriminating endoscopic features of SSL. These findings could enhance the detection of SSL in endoscopy practice.

\section{IDDF2021-ABS-0027 POST OPERATIVE PNEUMONIA AFTER ABDOMINAL SURGERY AND THE ROLE ON UNFAVORABLE OUTCOME: EVALUATION ON EMERGENCY ABDOMINAL SURGERY PATIENTS WITH COVID-19}

${ }^{1}$ Ricky Dwi Nur Tyastono*, ${ }^{2}$ Budhi Ida Bagus, ${ }^{1}$ Riza Setya Agrensa, ${ }^{1}$ Nugraha Muhammad Singgih. 'Department of Surgery, Moewardi General Hospital, Indonesia; ${ }^{2}$ Department of Surgery, Sebelas Maret University, Indonesia

\subsection{6/gutjnl-2021-IDDF.112}

Background Abdominal surgery is one of the most common procedures in a non-elective setting. Especially in the era of the COVID-19 pandemic nowadays, many kinds of abdominal surgery have been rescheduled and we must make priorities of what procedures should be done during the pandemic. Many guidelines (such as Royal College Surgery) suggested to postpone and reschedule the operative treatment during the pandemic, even in gastrointestinal cancer surgery. In this pandemic era, those guidelines could be applied in many elective settings, but not in emergency abdominal surgery procedures, we must face increasing post operative morbidity and mortality on performing surgery in confirmed COVID-19 will evaluate the post operative morbidity which leads to increased mortality of COVID-19 patients whose underwent emergency abdominal surgery during the pandemic.

Methods This study has conducted at Digestive Surgery Division at Moewardi General Hospital, Indonesia, from April 2020 until March 2021. Emergency abdominal surgery, including trauma and non-trauma cases, will be included in this study. All patients have already confirmed with positive COVID-19 pre-operatively. The procedure of this emergency surgery is laparotomy exploration with standardized level 3 PPE during surgery. The exclusion criteria of this study are to redo surgery and pediatric surgery case. The presence of post operative morbidity and in-hospital mortality will be recorded following this study.

Results From April 2020 until March 2021, we reported 39 emergency abdominal surgery cases. All of them have been done emergency exploratory laparotomy. The most common procedure is diffuse peritonitis due to peptic ulcer perforation (reported in 21 cases), other procedures are complicated appendicitis in 9 cases, two cases of blunt abdominal trauma, large bowel obstruction was found in 4 cases and 3 cases of incarcerated groin hernia. Thirty-one patients survived following the emergency abdominal surgery and 8 patients did not survive with post operative pneumonia and uncontrolled sepsis in the ICU.

Conclusions Post operative pneumonia is the important factor which has an impact on unfavorable clinical outcome during emergency abdominal surgery procedures on confirmed COVID-19 positive patients. These high-risk procedures need the perioperative multidiscipline team to gain a better outcome in this current pandemic.

\section{IDDF2021-ABS-0035 OPIUM ADDICTION INDUCED GASTRIC OUTLET OBSTRUCTION- AN UNUSUAL ENTITY}

Sudhir Maharshi*, Shyam Sunder Sharma. SMS Medical College and Hospitals, Jaipur, India

\subsection{6/gutjnl-2021-IDDF.113}

Background There is a paucity of data in the literature on opium addiction causing gastric outlet obstruction. The aim was to study opium addiction causing gastric outlet obstruction and to assess the role of endotherapy.

Methods Prospective study was conducted at SMS Hospital, Jaipur, between January 2017 to December 2020. All patients with a clinical diagnosis of gastric outlet obstruction with a history of opium addiction were consecutively enrolled. Data relating to demography, laboratory parameters, radiological imaging, histology and outcome of endotherapy were analyzed.

Results Sixty patients (age $38.3 \pm 11.2$ years, BMI $17.6 \pm 2.9 \mathrm{~kg}$ / $\mathrm{m}^{2}, 57$ males) were studied. Site of involvement was pylorus with junction of first and second part of duodenum in 24 $(40 \%)$, junction of first and second part of duodenum in 16 (26.7\%), pylorus with distal second part or third part of duodenum in $11(18.3 \%)$ as shown in figure $1 \& 2$ and pylorus, junction of first and second part of duodenum along with distal second part of duodenum or third part seen in $9(15 \%)$ patients (IDDF2021-ABS-0035 Figure 1. Endoscopic image showing ulcerated stricture at pylorus, IDDF2021-ABS-0035 Figure 2. Endoscopic image showing stricture in distal second

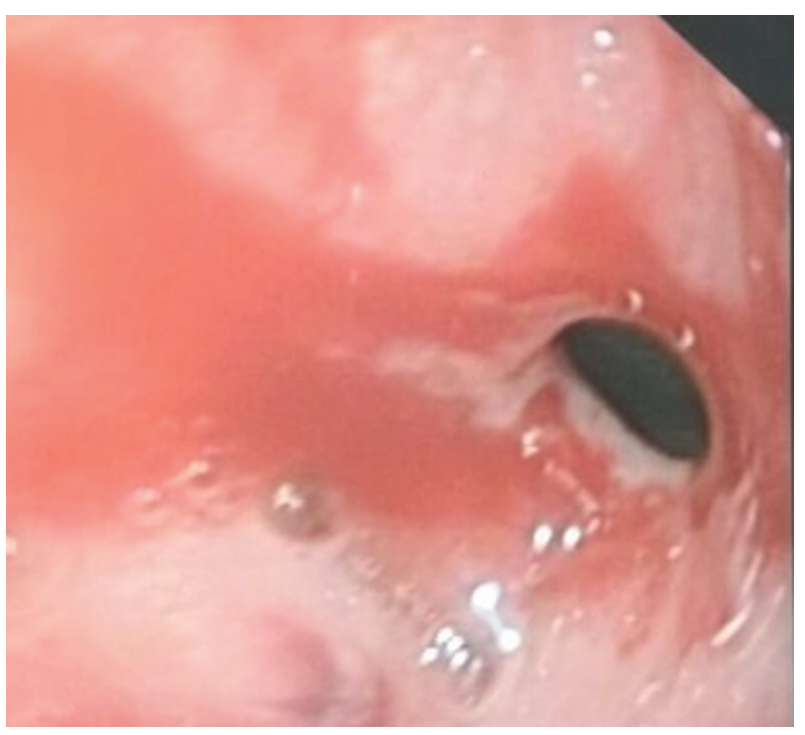

Abstract IDDF2021-ABS-0035 Figure 1 


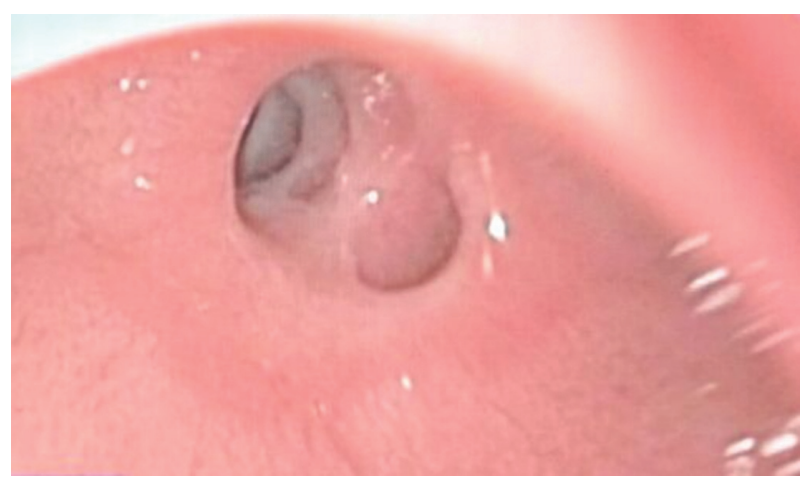

Abstract IDDF2021-ABS-0035 Figure 2

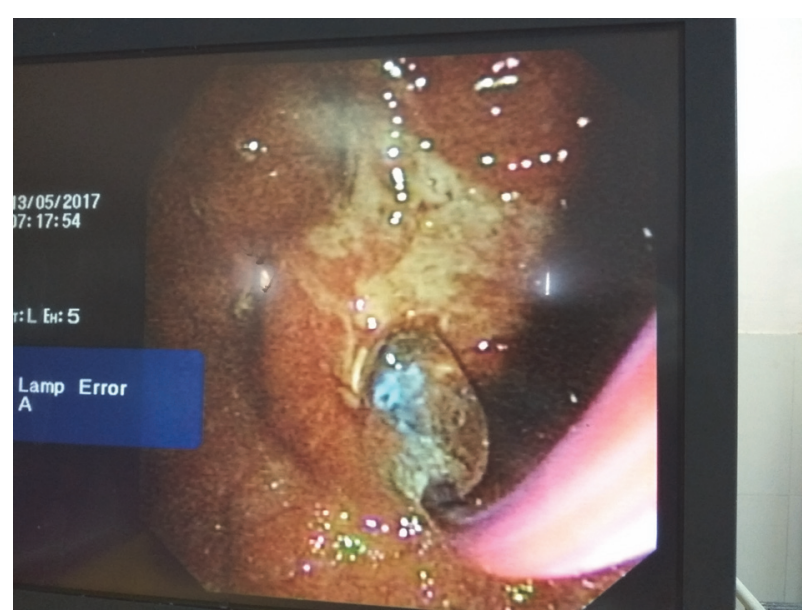

Abstract IDDF2021-ABS-0035 Figure 3

part of duodenum). The mean number of opium-containing analgesic tablets per patient was $5.2 \pm 3.1$ per day for duration of $33.1 \pm 25.8$ months. Vomiting (96.1\%), abdominal pain (93.5\%) and weight loss (85.2\%) were the most common presenting features. Forty-three $(71.6 \%)$ patients improved with endotherapy along with strict opium abstinence as shown in figure 3 while $17(28.3 \%)$ patients required surgical treatment (IDDF2021-ABS-0035 Figure 3. Endoscopic dilatation of pyloric stricture).

Conclusions Opium addiction is also a cause of benign gastric outlet obstruction. Gut involvement is seen on multiple sites, with most common at the junction of the first and second part of the duodenum. A successful outcome of endotherapy is more likely in these patients.

\section{IDDF2021-ABS-0036 PROGNOSTIC VALUE OF THE CEA, RDW- CV, RDW-SD COMBINED WITH CIP2A IN THE SERUM OF PATIENTS WITH COLORECTAL CANCER}

${ }^{1}$ Wei Chen*, ${ }^{2}$ Xiang Peng. 'Department of Colorectal Surgery, Guangdong Provincial Key Laboratory of Colorectal and Pelvic Floor Disease, The Sixth Affiliated Hospital, Sun Yat-sen University, China; 'Gastroenterology, Guangdong Provincial Key Laboratory of Colorectal and Pelvic Floor Disease, The Sixth Affiliated Hospital, Sun Yat-sen University, China

10.1136/gutjnl-2021-IDDF.114
Background To investigate the prognostic value of CEA, RDW-CV, RDW-SD and CIP2A in the serum of colorectal cancer (CRC) patients after resection.

Methods The clinical and follow-up data of 61 CRC patients who underwent surgery in the sixth Affiliated Hospital of Sun Yat-sen University January 2012 and December. The association between the CIP2A, CEA, RDW-CV, RDW-SD and the prognosis was evaluated. The Kaplan-Meier curves were used to identify the overall survival (OS) rates of the different groups.

Results At the end of the follow-up period (2020), the median follow-up is 207 months (range, 17-260 months). The 5-year OS of patients with low level of serum CIP2A was not better than that of high level, which is contrary to other indicators (CEA, RDW-CV and RDW-SD). Cox multivariate analysis showed that the age, $\mathrm{N}$ stage and $\mathrm{M}$ stage were independent factors influencing patient survival. The CRRC (combination of CEA, RDW-CV, RDW-SD and CIP2A) has better prognostic power than other indicators (CIP2A, CEA, RDW-CV and RDW-SD) according to the receiver operating characteristic (ROC) curve analysis.

Conclusions CRRC could be used as a better prognostic biomarker for CRC than the CEA, RDW-CV, RDW-SD and CIP2A alone.

\section{IDDF2021-ABS-0037 PROSPECTIVE EVALUATION OF AMOXICILLIN-CLARITHROMYCIN TRIPLE THERAPY IN PRIVATE CLINICAL PRACTICE IN HONG KONG FROM 2008 TO 2020}

Natalie Ning Yan Choi*, Sabrina Zi Yi Tee. Barts and the London, UK

\subsection{6/gutjnl-2021-IDDF.115}

Background There is a scarcity of local data regarding the current effectiveness of standard triple therapy in the treatment of Heliobacter pylori infection. To address the rising trend of bacterial resistance to antibiotics, we aim to monitor the efficacy of clarithromycin-containing triple therapy as a first-line treatment in Hong Kong and to assess the need for a longer treatment duration.

Methods 440 patients with a positive rapid urease test in oesophago-gastro-duodenoscopy (OGD) received clarithromycin-based triple therapy for a period of 7, 10 or 14 days according to treatment guidelines in the corresponding period of time. Patient demographics, indications for endoscopy and endoscopic diagnoses are detailed in Table 1 (IDDF2021-ABS0037 Table 1). Either a 7-day or 10-day treatment course was given between the period of 2008-2013, while a 10-day course was given from 2014-2015 and a 14-day course was given from 2016 onwards. Data analysis was carried out with chi-squared tests.

Data presented as $\mathrm{n}(\%)$. Both diagnoses were counted for those with two diagnosis, thus percentage total may exceed $100 \%$. Other indications for endoscopy included anorexia, epigastric discomfort, globus in throat, odynophagia, weight loss, dysphagia, hiccup, raised CA72.4, raised CEA, tarry stool, and those who were asymptomatic. Other endoscopic diagnoses included oesophageal ulcer, oesophagitis, gastric polyp, duodenitis, and oesophageal squamous dysplasia. 